Contemporary Mathematics, AMS series

Volume 211, 1997, 319-341

\title{
On Rigidity of One-Dimensional Maps
}

\author{
Yunping Jiang
}

\begin{abstract}
The regularity of the conjugacy between two onedimensional maps with singular points is considered. We prove that the conjugacy between two nice and mixing quasi-hyperbolic one-dimensional maps is a diffeomorphism if it is an absolutely continuous homeomorphism and the exponents and the asymmetries of these two maps at all corresponding singular points are the same. We also discuss the application to geometrically finite one-dimensional maps, to Ulam-von Neumann transformations, and to circle expanding maps.
\end{abstract}

\section{Introduction}

A well-recognised program in the study of dynamical systems is to "fill in" the dictionary between the theory of one-dimensional dynamical systems and the theory of Kleinian groups (see the papers of, among others, Bowen [BO], Shub and Sullivan [SS], Thurston [TH], McMullen [MC1,MC2], Gardiner and Sullivan [GS1,GS2], and Sullivan [SU1]). In Kleinian groups, a remarkable result is Mostow's rigidity theorem $[\mathbf{M O}]$. The theorem says that if two closed hyperbolic $n$-manifolds for $n \geq 3$ are homeomorphic, then they must be isometric. A closed hyperbolic $n$-manifold $M^{n}$ can be treated as the quotient space $M^{n}=\mathbb{H}^{n} / \Gamma$ of a Kleinian group $\Gamma$ which preserves the upper-half space $\mathbb{H}^{n}$. For $n=2$, Mostow's rigidity theorem can

1991 Mathematics Subject Classification. Primary 57F25, Secondary 58F11.. Partially supported by NSF grants and PSC-CUNY awards.

(C) American Mathematical Society $0271-4132 / 00 \$ 1.00+\$ .25$ per page 
be stated as follows. Let $\mathbb{D}$ be the open unit disk. A Kleinian group $\Gamma$ acting on $\mathbb{R}^{2}$ is called Fuchsian if it preserves $\mathbb{D}$. A Fuchsian group $\Gamma$ is called co-compact if the quotient space $\mathcal{S}=\mathbb{D} / \Gamma$ is a closed hyperbolic Riemann surface. Suppose $\Gamma_{1}$ and $\Gamma_{2}$ are two isomorphic co-compact Fuchsian groups. Then there is a homeomorphism $H$ of $\overline{\mathbb{D}}$ such that

$$
H \Gamma_{1}=\Gamma_{2} H .
$$

Let $\mathbb{S}^{1}$ be the unit circle which is the boundary of $\mathbb{D}$. Mostow's rigidity theorem in this case says that if $H \mid \mathbb{S}^{1}$ is an absolutely continuous homeomorphism, then $H$ must be a Möbius transformation. A corresponding result in the theory of one-dimensional dynamics was proved by Shub and Sullivan [SS]. Their result can be described as follows: let $f$ be a $C^{2}$ orientation-preserving endomorphism of the circle $\mathbb{S}^{1}$. It is said to be expanding if there are constants $C>0$ and $\lambda>1$ such that $\left|\left(f^{\circ n}\right)^{\prime}\right| \geq C \lambda^{n}$ for all $x$ in $\mathbb{S}^{1}$ and all $n \geq 1$. Shub [SH] proved that any $C^{2}$ orientation-preserving expanding endomorphism $f$ of $\mathbb{S}^{1}$ is topologically conjugate to $z \mapsto z^{d}$ on $\mathbb{S}^{1}$ for a unique integer $d \geq 2$, where $d$ is called the degree of $f$. Therefore, any two $C^{2}$ orientation-preserving circle endomorphisms $f$ and $g$ with the same degree is topologically conjugate. Let $h$ be the conjugacy from $f$ to $g$, i.e.,

$$
h \circ f=g \circ h .
$$

Shub and Sullivan [SS] proved that if both $f$ and $g$ are $C^{k}$ for some $k \geq 2$ and if $h$ is an absolutely continuous homeomorphism, then $h$ must be also a $C^{k}$ diffeomorphism. We would like to note that there is a proof of Mostow's rigidity theorem from dynamical system point of view (see $[\mathbf{B O}]$ ) by considering the induced expanding Markov maps $r_{1}$ and $r_{2}$ of $\mathbb{S}^{1}$ such that $h \circ r_{1}=r_{2} \circ h$ for $h=H \mid \mathbb{S}^{1}$.

Mostow's rigidity theorem is generalised for a bigger class of Fuchsian groups $\Gamma$ such that the quotient space $\mathcal{S}=\mathbb{D} / \Gamma$ has finite hyperbolic volume (see, for example, [MO,TU,TH,SU2]). The corresponding bigger class in one-dimensional dynamics should be the class of one-dimensional dynamical systems having critical points and having certain mixing property. The first corresponding result in the bigger class is proved in $[\mathbf{J I 1}, \mathbf{J I} 4]$ for generalised Ulam-von Neumann transformations which are certain interval maps with one power law type critical point (where we used the Gibbs theory in the study of this problem). Other corresponding results in the bigger class are developed in $[\mathbf{J I 3}, \mathbf{J I 5}]$ later for geometrically finite onedimensional maps. (In [JI5], the scaling function for a geometrically 
finite one-dimensional map is defined, studied, and used as a complete smooth invariant along with exponents and asymmetries at all singular points). However, the maps considered in [SS,JI4,JI5] are all geometrically finite from the combinatorics point of view. In this paper, we further develop our method in $[\mathbf{J I 1}, \mathbf{J I 2}, \mathbf{J I 3}, \mathbf{J I 4}, \mathbf{J I 5}]$ to a space including some geometrically infinite one-dimensional dynamical systems.

The paper is organised as follows. In $\S 2$, we gave the definition of a quasi-hyperbolic one-dimensional map (Definition 2), which is certain one-dimensional map with non-recurrent critical points. One of the main technique tools [JI2] in our study is discussed in $\S 3$. This technique tool (Lemma 2) estimates the nonlinearity of the dynamical system generated by a quasi-hyperbolic one-dimensional map. We also define the nice and mixing conditions for a quasi-hyperbolic one-dimensional map in the beginning of $\S 4$. Let $S P$ be the set of all singular points of a quasi-hyperbolic one-dimensional map $f$, let $P S O=\cup_{i=1}^{\infty} f^{\circ i}(S P)$ be the set of post-singular orbits of $f$, let $\overline{P S O}$ be the closure of $P S O$. A point $p$ in $S P$ is fold if $f^{\prime}(x) / f^{\prime}(y)<0$ for points $x<p<y$ close to $p$. Let $F S P$ be the set of all fold singular points of $f$ and let $N F S P=S P \backslash F S P$ be the set of all non-fold singular points of $f$. Let $P N F S O=\cup_{n=1}^{\infty} f^{\circ n}(N F S P)$ be the set of post non-fold singular orbits of $f$. Let $\Gamma=\overline{P S O} \backslash P N F S O$ and $M_{0}=M \backslash \Gamma$. Let $\eta$ be the set of closures of intervals of $M_{0}$. In $\S 4$, we prove that

MAIn TheOREM. Suppose $f$ and $g$ are conjugate nice and mixing quasi-hyperbolic one-dimensional maps. Suppose the conjugacy $h$ from $f$ to $g$, i.e., $h \circ f=g \circ h$, is orientation-preserving homeomorphism of $M$. The map $h$ restricted to every interval $J$ in $\eta$ is a $C^{1+\beta}$ for some fixed $0<\beta \leq 1$ diffeomorphism if

a $h$ is an absolutely continuous homeomorphism and

$\mathbf{b}$ the exponents and the asymmetries of $f$ and $g$ at all corresponding singular points are the same.

In $\S 5, \S 6$, and $\S 7$, we discuss some application of the main result to geometrically finite one-dimensional maps (Corollary 1), to generalised Ulam-von Neumann transformations (Corollaries 2 and 3), and to expanding circle endomorphisms (Corollary 4).

Acknowledgment. The author would like to thank Professor Dennis Sullivan for insightful suggestions. 


\section{Sub-hyperbolic one-dimensional maps}

Let $M$ be the interval $[-1,1]$ or the unit circle $\mathbb{S}^{1}=\mathbb{R} / \mathbb{Z}$. Let $f: M \rightarrow M$ be a piecewise $C^{1}$ map. A point $c \in M$ is said to be singular if either $f^{\prime}(c)$ does not exist or $f^{\prime}(c)$ exists but $f^{\prime}(c)=0$. The later one is called a critical point. A singular point $c$ of $f$ is said to be power law if there is a real number $\gamma \geq 1$ such that the limits

$$
\lim _{x \rightarrow c-} \frac{f^{\prime}(x)}{|x-c|^{\gamma-1}}=B_{-} \quad \text { and } \quad \lim _{x \rightarrow c+} \frac{f^{\prime}(x)}{|x-c|^{\gamma-1}}=B_{+}
$$

exist and are non-zero. The numbers $\gamma$ and $A=B_{-} / B_{+}$are called the exponent and asymmetry of $f$ at $c$. Let

$$
r_{c}(x)=\frac{f^{\prime}(x)}{|x-c|^{\gamma-1}}
$$

for any power law singular point $c$.

REMARK 1 . The exponent and the asymmetry of $f$ at a power law singular point are (orientation) $C^{1}$-invariant, this means that if $h \circ f=g \circ h$ for an orientation-preserving $C^{1}$ diffeomorphism $h$, then the exponents and the asymmetries of $f$ and $g$ are the same at corresponding power law singular points.

Henceforth, we assume that $f: M \rightarrow M$ has only power law singular points and the number of power law singular points is finite (could be zero). Let $S P$ denote the set of all singular points of $f$ and let $C P$ denote the set of all critical points of $f$. Let $P S O=$ $\cup_{i=1}^{\infty} f^{\circ i}(S P)$ be the set of post-singular orbits. For a critical point $c \in C P$ and a real number $\tau>0$, let $U_{c}(\tau)=[c-\tau, c+\tau]$ and let $U(\tau)=\cup_{c \in C P} U_{c}(\tau)$. Let $V(\tau)=\overline{M \backslash U(\tau / 2)}$. A sequence of intervals $\left\{I_{i}\right\}_{i=0}^{n}$ is said to be a chain if $I_{i} \subset M \backslash S P$ and if $f: I_{i} \rightarrow I_{i+1}$ is bijective for all $i=0,1, \ldots, n-1$.

Definition 1 . We say a map $f: M \rightarrow M$ is $C^{1+}$ if there is a real number $0<\alpha \leq 1$ such that

(1) $f^{\prime}$ is $\alpha$-Hölder continuous when restricted to every interval in $M \backslash S P$ and

(2) there is a real number $\tau>0$ such that for every $c \in C P$, $r_{c}$ is $\alpha$-Hölder when restricted to $U_{c}(\tau) \cap\{x<c\}$ and to $U_{c}(\tau) \cap\{x>c\}$. 
Definition 2. A $C^{1+} \operatorname{map} f: M \rightarrow M$ is said to be quasihyperbolic if there is a constant $\tau>0$ such that $\overline{P S O} \cap U(\tau)=\emptyset$ and such that there exist constants $C>0$ and $0<\lambda<1$ satisfying that for any chain $\left\{I_{i}\right\}_{i=0}^{n}$, if either

(i) $I_{i} \subseteq V(\tau)$ for all $0 \leq i \leq n-1$ or

(ii) $I_{n} \subseteq U(\tau)$, then

$$
\left|I_{0}\right| \leq C \lambda^{n}\left|I_{n}\right|
$$

The space of quasi-hyperbolic one-dimensional maps contains many interesting maps. To show this let us give some examples. A point $q$ in $M$ is called periodic of period $k$ of $f$ if $f^{\circ i}(q) \neq q$ for all $0<i<k$ but $f^{\circ k}(q)=q$. For a periodic point $q$ of period $k$ of $f$, let $O=\left\{f^{\circ i}(p)\right\}_{i=0}^{k-1}$ be the periodic orbit and let $E_{q, f}=\left(f^{\circ k}\right)^{\prime}(q)$ be the eigenvalue of $f$ at $O$ (or at $q$ ). The periodic orbit $O$ is called attractive if $\left|E_{q, f}\right|<1$; parabolic if $\left|E_{q, f}\right|=1$; expanding if $\left|E_{q, f}\right|>1$. The first example comes from a theorem (see [MV, Theorem 6.3, pp. 261-262]). A critical point $c$ of a $C^{2}$ map is called non-degenerate if $f^{\prime}(c)=0$ and if $f^{\prime \prime}(c) \neq 0$.

ExAmple 1. A $C^{2}$ map $f: M \rightarrow M$ with only non-degenerate critical points such that $\overline{P S O} \cap S P=\emptyset$ and such that all periodic points are expanding.

The Schwarzian derivative of a $C^{3}$ map $f: M \rightarrow M$ is, by definition,

$$
S(f)=\frac{f^{\prime \prime \prime}}{f^{\prime}}-\frac{3}{2}\left(\frac{f^{\prime \prime}}{f^{\prime}}\right)^{2} .
$$

A $C^{3}$ map $f: M \rightarrow M$ has negative Schwarzian derivative if $S(f)(x)<$ 0 for all $x$ in $M$. Singer (see [MV]) proved that if $f$ has negative Schwarzian derivative, then the immediate basin of every attractive or parabolic periodic orbit contains at least one critical orbit. Therefore, if $f$ has negative Schwarzian derivative, if $\overline{P S O} \cap S P=\emptyset$, and if $\overline{P S O}$ contains neither attractive nor parabolic periodic points, then all periodic points of $f$ are expanding. We then have that

ExAmple 2. A $C^{3}$ map $f: M \rightarrow M$ having negative Schwarzian derivative and satisfying that $\overline{P S O} \cap S P=\emptyset$ and that $\overline{P S O}$ contains neither attractive periodic points nor parabolic periodic points.

Suppose $f: M \rightarrow M$ is a $C^{3}$ map. If $f$ satisfies the condition that for every critical point $c, p=f^{\circ m}(c)$ is an expanding periodic point of $f$ for some integer $m \geq 1$, then $\overline{P S O}=P S O$ contains neither 
attractive nor parabolic periodic points. The condition here is called the preperiodic condition. In particular, Example 2 says that a $C^{3}$ map $f: M \rightarrow M$ having negative Schwarzian derivative and satisfying the preperiodic condition is quasi-hyperbolic.

There are two more examples. One is a $C^{1+\alpha}$ circle expanding map where $0<\alpha \leq 1$ (see $\S 7$ ). The other is a geometrically finite onedimensional map (see $\S 5$ and $[\mathbf{J I 3}, \mathbf{J I 5}]$ ). In the space of geometrically finite one-dimensional maps, a more interesting example is a generalised Ulam-von Neumann transformation (see $\S 6$ and $[\mathbf{J I 1}, \mathbf{J I} 4]$ ). The definitions of these maps are given in $\S 5, \S 6$, and $\S 7$, respectively. We also discuss some applications of the Main Theorem to these maps in these sections.

\section{Distortion of a quasi-hyperbolic map}

Suppose $f: M \rightarrow M$ is a quasi-hyperbolic map. Let $C P=$ $\left\{c_{1}, \cdots, c_{d}\right\}$ be the set of all critical points of $f$ (it may be empty) and let $\Gamma=\left\{\gamma_{1}, \cdots, \gamma_{d}\right\}$ be the set of corresponding exponents. Let $\tau>0$ be a fixed number satisfying Definitions 1 and 2. Let $U_{i}(\tau)=$ $\left[c_{i}-\tau, c_{i}+\tau\right]$ and let

$$
U=U(\tau)=\cup_{i=1}^{d} U_{i}(\tau) .
$$

Let $U_{i}(\tau / 2)=\left[c_{i}-\tau / 2, c_{i}+\tau / 2\right]$, let $\tilde{U}=\cup_{i=1}^{k} U_{i}\left(\frac{\tau}{2}\right)$, and let

$$
V=\overline{M \backslash \tilde{U}}
$$

Note that $U$ and $V$ are closed sets. They are fixed in the rest of the paper.

Dividing the space of $M$ into $U$ and $V$ is one of the key points in the paper: the set $V$ is away from all critical points $C P$ and the set $U$ is away from post-singular orbit $P S O=\cup_{n=1}^{\infty} f^{\circ n}(S P)$. In the set $V$ we can use the naive distortion property to control the distortion of the iterates of $f$ (Lemma 1), and in the set $U$ we can prove a Koebe type distortion property to control the distortion of the iterates of $f$ (Lemma 2).

Let $0<\alpha \leq 1$ be a number in Definition 1 satisfied by $f$. A chain $\mathcal{I}=\left\{I_{i}\right\}_{i=0}^{n}$ of closed intervals of $M$ is said to be admissible if $f: I_{i} \rightarrow I_{i+1}$ is a $C^{1+\alpha}$-diffeomorphism and if either $I_{i} \subseteq V$ or $I_{i} \subseteq U$ for all $0 \leq i \leq n$. For any $x$ in $M$, let $x_{n}=f^{\circ n}(x)$.

LEMMA 1. There is a constant $C>0$ such that for any admissible chain $\mathcal{I}=\left\{I_{i}\right\}_{i=0}^{n}$ if $I_{i} \subseteq V$ for all $1 \leq i \leq n-1$, then for any $x$ and 
$y$ in $I_{0}$

$$
\left|\log \left(\frac{\left|\left(f^{\circ}\right)^{\prime}(x)\right|}{\left|\left(f^{\circ}\right)^{\prime}(y)\right|}\right)\right| \leq C\left|x_{n}-y_{n}\right|^{\alpha} .
$$

Proof. Let $\xi$ be the set of the closures of intervals in $V \backslash S P$. Let

$$
a_{0}=\inf _{x \in V \backslash S P}\left|f^{\prime}(x)\right|>0
$$

and let

$$
b_{0}=\sup _{x \neq y \in I, I \in \xi} \frac{\left|f^{\prime}(x)-f^{\prime}(y)\right|}{|x-y|^{\alpha}}<\infty .
$$

For any $x$ and $y$ in $I_{0}$, let $x_{i}=f^{\circ i}(x)$ and $y_{i}=f^{\circ i}(y)$ for $0 \leq i \leq n$. Then

$$
\mathcal{A}=\frac{\left|\left(f^{\circ n}\right)^{\prime}(x)\right|}{\left|\left(f^{\circ n}\right)^{\prime}(y)\right|}=\prod_{i=0}^{n-1} \frac{\left|f^{\prime}\left(x_{i}\right)\right|}{\left|f^{\prime}\left(y_{i}\right)\right|} .
$$

This implies that

$$
|\log \mathcal{A}| \leq \frac{b_{0}}{a_{0}} \sum_{i=0}^{n-1}\left|x_{i}-y_{i}\right|^{\alpha}
$$

From Definition 2, there are constants $C_{0}>0$ and $0<\lambda_{0}<1$ such that

$$
\left|x_{i}-y_{i}\right| \leq C_{0} \lambda_{0}^{n-i}\left|x_{n}-y_{n}\right|
$$

for $0 \leq i \leq n-1$. Therefore,

$$
|\log \mathcal{A}| \leq \frac{b_{0} C_{0}}{a_{0}\left(1-\lambda_{0}^{\alpha}\right)}\left|x_{n}-y_{n}\right|^{\alpha}=C\left|x_{n}-y_{n}\right|^{\alpha}
$$

where $C=\left(b_{0} C_{0}\right) /\left(a_{0}\left(1-\lambda_{0}^{\alpha}\right)\right)$.

The following lemma is one of the key lemmas (refer to [JI2]) and is a property similar to Koebe's distortion theorem in one complex variable.

LEMMA 2. There is a constant $C>0$ such that for any admissible chain $\mathcal{I}=\left\{I_{i}\right\}_{i=0}^{n}$ if $I_{n} \subset U$, then for any $x$ and $y$ in $I_{0}$,

$$
\left|\log \left(\frac{\left|\left(f^{\circ n}\right)^{\prime}(x)\right|}{\left|\left(f^{\circ n}\right)^{\prime}(y)\right|}\right)\right| \leq C\left|x_{n}-y_{n}\right|^{\alpha} .
$$


Proof. The ratio $\left|\left(f^{\circ n}\right)^{\prime}(x)\right| /\left|\left(f^{\circ n}\right)^{\prime}(y)\right|$ equals the product

$$
\mathcal{A}=\prod_{i=0}^{n-1} \frac{\left|f^{\prime}\left(x_{i}\right)\right|}{\left|f^{\prime}\left(y_{i}\right)\right|}
$$

where $x_{i}=f^{\circ i}(x)$ and $y_{i}=f^{\circ i}(y)$ for $0 \leq i \leq n$. Let $\left(x_{i}, y_{i}\right)$ mean the open interval in $I_{i}$ bounded by $x_{i}$ and $y_{i}$. We divide the set of intervals $\mathcal{J}=\left\{\left(x_{i}, y_{i}\right)\right\}_{i=0}^{n-1}$ into two subsets

$$
\mathcal{J}_{1}=\left\{\left(x_{i}, y_{i}\right) \mid\left(x_{i}, y_{i}\right) \subseteq V\right\} \text { and } \mathcal{J}_{2}=\left\{\left(x_{i}, y_{i}\right) \mid\left(x_{i}, y_{i}\right) \subseteq U \backslash V\right\} .
$$

Consider

$$
\prod_{x_{i}, y_{i} \in \mathcal{J}_{1}} \frac{\left|f^{\prime}\left(x_{i}\right)\right|}{\left|f^{\prime}\left(y_{i}\right)\right|} \quad \text { and } \quad \prod_{x_{i}, y_{i} \in \mathcal{J}_{2}} \frac{\left|f^{\prime}\left(x_{i}\right)\right|}{\left|f^{\prime}\left(y_{i}\right)\right|}
$$

Following the proof of Lemma 1 , there are constants $C_{1}, C_{2}>0$ such that

$$
\left|\log \left(\prod_{x_{i}, y_{i} \in \mathcal{J}_{1}} \frac{\left|f^{\prime}\left(x_{i}\right)\right|}{\left|f^{\prime}\left(y_{i}\right)\right|}\right)\right| \leq C_{1} \sum_{x_{i}, y_{i} \in \mathcal{J}_{1}}\left|x_{i}-y_{i}\right|^{\alpha} \leq C_{2} \sum_{x_{i}, y_{i} \in \mathcal{J}_{2}}\left|x_{i}-y_{i}\right|^{\alpha} .
$$

To estimate the product $\prod_{x_{i}, y_{i} \in \mathcal{J}_{2}}\left|f^{\prime}\left(x_{i}\right)\right| /\left|f^{\prime}\left(y_{i}\right)\right|$, we write it as the product of three factors:

$$
\begin{gathered}
\mathcal{B}=\prod_{x_{i}, y_{i} \in \mathcal{J}_{2}}\left(\frac{\left|x_{i}-c_{k_{i}}\right|^{\gamma_{k_{i}}}}{\left|f\left(x_{i}\right)-f\left(c_{k_{i}}\right)\right|} \frac{\left|f\left(y_{i}\right)-f\left(c_{k_{i}}\right)\right|}{\left|y_{i}-c_{k_{i}}\right|^{\gamma_{k_{i}}}}\right)^{t_{k_{i}}}, \\
\mathcal{C}=\prod_{x_{i}, y_{i} \in \mathcal{J}_{2}} \frac{\left|y_{i}-c_{k_{i}}\right|^{\gamma_{k_{i}}-1}}{\left|f^{\prime}\left(y_{i}\right)\right|} \frac{\left|f^{\prime}\left(x_{i}\right)\right|}{\left|x_{i}-c_{k_{i}}\right|^{\gamma_{k_{i}}-1}},
\end{gathered}
$$

and

$$
\mathcal{D}=\prod_{x_{i}, y_{i} \in \mathcal{J}_{2}}\left(\frac{\left|f\left(x_{i}\right)-f\left(c_{k_{i}}\right)\right|}{\left|f\left(y_{i}\right)-f\left(c_{k_{i}}\right)\right|}\right)^{t_{k_{i}}}
$$

where $x_{i}$ and $y_{i}$ are in $U_{k_{i}}(\tau)$ and $t_{k_{i}}=\left(\gamma_{k_{i}}-1\right) / \gamma_{k_{i}}$. From Definition 1 and following the proof of Lemma 1 , there is a constant $C_{3}>0$ such that

$$
|\log \mathcal{B}|,|\log \mathcal{C}| \leq C_{3} \sum_{x_{i}, y_{i} \in \mathcal{J}_{2}}\left|x_{i}-y_{i}\right|^{\alpha}
$$

Now we concentrate on the estimate of $\mathcal{D}$. Let

$$
\frac{f\left(x_{i}\right)-f\left(c_{k_{i}}\right)}{f\left(y_{i}\right)-f\left(c_{k_{i}}\right)}=1+\frac{f\left(x_{i}\right)-f\left(y_{i}\right)}{f\left(y_{i}\right)-f\left(c_{k_{i}}\right)} .
$$


Then

$$
\mathcal{D}=\exp \left(\sum_{s=1}^{r-1} \frac{1}{t_{k_{i_{s}}}} \log \left|1+\frac{f\left(x_{i_{s}}\right)-f\left(y_{i_{s}}\right)}{f\left(y_{i_{s}}\right)-f\left(c_{k_{i_{s}}}\right)}\right|\right)
$$

where $i_{1}<i_{2}<\cdots<i_{r-1}<n$. Let $i_{r}=n$. For each $i_{s}, 1 \leq s<r$, consider the interval $L_{s}$ bounded by $y_{i_{s}}$ and $c_{k_{i_{s}}}$ and the map $h_{s}=$ $f^{\circ\left(i_{s+1}-i_{s}\right)}$. Let $R_{s} \subseteq L_{s}$ be the maximal interval containing $y_{i_{s}}$ such that $h_{s}$ on $R_{s}$ is $C^{1}$ and injective. One of the endpoints of $R_{s}$ is $y_{i_{s}}$ and the other is a preimage $e$ of a singular point $q$ in $S P$ under $f^{\circ k_{s}}$ for some $0 \leq k_{s}<i_{s+1}-i_{s}$. Let $l_{s}=i_{s+1}-i_{s}-k_{s}$. Then $h_{s}$ on the minimal interval $J_{s}$ containing $x_{i_{s}}$ and $R_{s}$ is injective and maps $J_{s}$ onto an interval containing the points $y_{i_{s+1}}, x_{i_{s+1}}$ and $f^{\circ l_{s}}(q)$. We enlarge every interval $J$ of $V$ into a closed interval $J^{\prime} \supset J$ such that $J^{\prime} \cap C P=\emptyset$ and such that the length of $J^{\prime} \cap U$ is greater than a constant $a>0$. Let $V^{\prime}=\cup_{J \in V} J^{\prime}$ be the union of all these enlarged intervals and let $U^{\prime}=M \backslash V^{\prime}$. If $f^{\circ i}\left(J_{s}\right) \subseteq V^{\prime}$ for all $1 \leq i<i_{s+1}-i_{s}$, by following the proof of Lemma 1 , there is a constant $C_{4}>0$ such that

$$
\frac{\left|f\left(x_{i_{s}}\right)-f\left(y_{i_{s}}\right)\right|}{\left|f\left(y_{i_{s}}\right)-f\left(c_{k_{i_{s}}}\right)\right|} \leq C_{4} \frac{\left|x_{i_{s+1}}-y_{i_{s+1}}\right|}{\left|y_{i_{s+1}}-f^{\circ l_{s}}(q)\right|} .
$$

Since $y_{i_{s+1}}$ is in $U$ and $f^{\circ l_{s}}(q)$ is in $P S O$,

$$
\frac{\left|f\left(x_{i_{s}}\right)-f\left(y_{i_{s}}\right)\right|}{\left|f\left(y_{i_{s}}\right)-f\left(c_{k_{i_{s}}}\right)\right|} \leq C_{4} \frac{\left|x_{i_{s+1}}-y_{i_{s+1}}\right|}{C_{0}}
$$

where $C_{0}>0$ is the distance between $U$ and $\overline{P S O}$. Otherwise, let $0<k<i_{s+1}-i_{s}$ be the smallest integer such that $f^{\circ k}\left(J_{s}\right) \cap U^{\prime} \neq \emptyset$. Since $f^{\circ i}\left(J_{s}\right) \subseteq V^{\prime}$ for all $1 \leq i<k$, following the proof of Lemma 1 , there is a constant $C_{5}>0$ such that

$$
\frac{\left|f\left(x_{i_{s}}\right)-f\left(y_{i_{s}}\right)\right|}{\left|f\left(y_{i_{s}}\right)-f\left(c_{k_{i_{s}}}\right)\right|} \leq C_{5} \frac{\left|x_{i_{s}+k}-y_{i_{s}+k}\right|}{\left|y_{i_{s}+k}-f^{\circ k}(e)\right|} .
$$

Since $y_{i_{s}+k}$ is in $V$ and $f^{\circ k}(e)$ is in $U^{\prime}$,

$$
\frac{\left|f\left(x_{i_{s}}\right)-f\left(y_{i_{s}}\right)\right|}{\left|f\left(y_{i_{s}}\right)-f\left(c_{k_{i_{s}}}\right)\right|} \leq C_{5} \frac{\left|x_{i_{s}+k}-y_{i_{s}+k}\right|}{C_{0}^{\prime}}
$$

where $C_{0}^{\prime}>0$ is the distance between $V$ and $U^{\prime}$. From Definition 2 again, there is a constant $C_{5}^{\prime}>0$ such that

$$
\left|x_{i_{s}+k}-y_{i_{s}+k}\right| \leq C_{5}^{\prime}\left|x_{i_{s+1}}-y_{i_{s+1}}\right| \text {. }
$$


Hence, there is a constant $C_{6}>0$ such that

$$
|\log \mathcal{D}| \leq C_{6} \sum_{x_{i}, y_{i} \in \mathcal{J}_{2}}\left|x_{i}-y_{i}\right|^{\alpha} .
$$

Combining all the estimates, we have a constant $C_{7}>0$ such that

$$
\left|\log \left(\frac{\left|\left(f^{\circ n}\right)^{\prime}(x)\right|}{\left|\left(f^{\circ n}\right)^{\prime}(y)\right|}\right)\right| \leq C_{7} \sum_{x_{i}, y_{i} \in \mathcal{J}_{2}}\left|x_{i}-y_{i}\right|^{\alpha} .
$$

Applying ( $i i)$ of Definition 2, there are two constants $C_{8}>0$ and $0<\lambda<1$ such that

$$
\left|x_{i}-y_{i}\right| \leq C_{8} \lambda^{n-i}\left|x_{n}-y_{n}\right| .
$$

Therefore, we have a constant $C>0$ such that

$$
\left|\log \left(\frac{\left|\left(f^{\circ n}\right)^{\prime}(x)\right|}{\left|\left(f^{\circ n}\right)^{\prime}(y)\right|}\right)\right| \leq C\left|x_{n}-y_{n}\right|^{\alpha} .
$$

\section{Conjugacies between} quasi-hyperbolic one-dimensional maps

Suppose $f: M \rightarrow M$ is a quasi-hyperbolic one-dimensional map. Remember that $S P$ is the set of all singular points of $f$, that $P S O=$ $\cup_{i=1}^{\infty} f^{\circ i}(S P)$ is the set of post-singular orbits of $f$, and that $\overline{P S O}$ is the closure of $P S O$. If $\overline{P S O}$ occupies too much space in $M$, it will be difficult to have a nice result. To avoid this, we will only consider a nice quasi-hyperbolic one-dimensional map. Let $m$ be the Lebesgue measure on $M$. A quasi-hyperbolic one-dimensional map is said to be nice if

(I) $m(\overline{P S O})=0$ and

(II) there is an open neighborhood $W$ about $\overline{P S O}$ (i.e., $\overline{P S O} \subset W$ ) such that $M \backslash W$ is non-empty and such that for any point $p$ in $M$ either $\left\{f^{\circ n}(p)\right\}_{n=N}^{\infty} \subseteq \overline{P S O}$ for some $N>0$ or there is a subsequence $\left\{f^{\circ n_{i}}(p)\right\}_{i=1}^{\infty} \subseteq M \backslash W$.

We also need the mixing condition on $f$ so that $\left\{f^{\circ n}\right\}_{n=0}^{\infty}$ can not be decomposed into several dynamical systems. We say that $f$ is mixing if for any intervals $I$ and $J$ of $M$, there is an integer $n \geq 0$ such that $f^{\circ n}(J) \supseteq I$. The mixing condition is topologically invariant. 
A point $p$ in $S P$ is fold if the asymmetry $A_{p, f}$ of $f$ at $p$ is negative. (It is equivalent to say that $p$ is a local maximum or minimum point or to say that $f^{\prime}(x) / f^{\prime}(y)<0$ for points $x<p<y$ close to $p$.) Let $F S P$ be the set of all fold singular points of $f$ and let $N F S P=S P$ $F S P$ be the set of all non-fold singular points of $f$. Let $P N F S O=$ $\cup_{n=1}^{\infty} f^{\circ n}(N F S P)$ be the set of post non-fold singular orbits of $f$. Let $\Gamma=\overline{P S O} \backslash P N F S O$ and $M_{0}=M \backslash \Gamma$. Let $\eta$ be the set of closures of intervals of $M_{0}$. A bijective map $h: M \rightarrow M$ is called an absolutely continuous homeomorphism if $h$ and $h^{-1}$ are absolutely continuous.

Main Theorem. Suppose $f$ and $g$ are conjugate nice and mixing quasi-hyperbolic one-dimensional maps. Suppose the conjugacy $h$ from $f$ to $g$, i.e., $h \circ f=g \circ h$, is orientation-preserving homeomorphism of $M$. The map $h$ restricted to every interval $I$ in $\eta$ is a $C^{1+\beta}$ for some fixed $0<\beta \leq 1$ diffeomorphism if

a) $h$ is an absolutely continuous homeomorphism and

b) the exponents and the asymmetries of $f$ and $g$ at all corresponding singular points are the same.

Let $f$ and $g$ be maps and $h$ be the conjugacy in the theorem. Let $0<\alpha \leq 1$ be a number in Definition 1 satisfied by both $f$ and $g$. Let $C P=\left\{c_{1}, \cdots, c_{d}\right\}$ be the set of critical points of $f$. Let $\Gamma=\left\{\gamma_{1}, \cdots, \gamma_{d}\right\}$ be the set of corresponding exponents of $f$ at critical points. Let $\gamma=\max \left\{\gamma_{i} \mid 1 \leq i \leq d\right\}$.

REMARK 2. Then in the Main Theorem, $\beta=\alpha / \gamma$ if $h$ satisfies a) and b).

For any point $p$ in $M$, from the equation $h \circ f=g \circ h$, we have that if $p$ is not a singular point of $f$, then $h$ is differentiable at $p$ if and only if $h$ is differentiable at $f(p)$. Moreover, if $h$ is differentiable at $p$ and $f(p)$, we have

$$
h^{\prime}(p)=\frac{f^{\prime}(p)}{g^{\prime}(h(p))} h^{\prime}(f(p)) .
$$

REMARK 3. If $p$ is a singular point, let $\gamma_{f, p}$ and $\gamma_{g, h(p)}$ be the exponents of $f$ and $g$ at $p$ and at $h(p)$. Let $A_{f, p}=B_{-, f} / B_{+, f}$ and $A_{g, h(p)}=B_{-, g} / B_{+, g}$ be the asymmetry of $f$ and $g$ at $p$ and at $h(p)$. If $\gamma_{f, p}=\gamma_{g, h(p)}$ and if $h^{\prime}(f(p)) \neq 0$ exists, then

$$
\left(h^{\prime}(p-)\right)^{\gamma_{f, p}}=\frac{B_{-, f}}{B_{-, g}} h^{\prime}(f(p))
$$


and

$$
\left(h^{\prime}(p+)\right)^{\gamma_{f, p}}=\frac{B_{+, f}}{B_{+, g}} h^{\prime}(f(p))
$$

Furthermore, if $A_{f, p}=A_{g, h(p)}$, then $h^{\prime}(p-)=h^{\prime}(p+)$. Therefore, if $h^{\prime}(f(p))$ exists and if $\gamma_{f, p}=\gamma_{g, h(p)}$ and $A_{f, p}=A_{g, h(p)}$, then $h^{\prime}(p)$ exists. Similarly, if $h^{\prime}(p)$ exists, if $p$ is not a fold singular point, and if $\gamma_{f, p}=\gamma_{g, h(p)}$ and $A_{f, p}=A_{g, h(p)}$, then $h^{\prime}(f(p))$ exists.

Remember that $m$ is the Lebesgue measure on $M$. We say that a measurable set $E$ in an interval $I$ has full measure if $m(E)=m(I)$. A point $p$ in a measurable set $E_{0}$ is said to be a density point if

$$
\lim _{m(J) \rightarrow 0} \frac{m\left(E_{0} \cap J\right)}{m(J)}=1
$$

where $J$ runs over all intervals containing $p$. We prove the Main Theorem through several lemmas (Lemma 3 to Lemma 5).

LEMMA 3. If $h$ is absolutely continuous, then we can find a small interval $I \subset M \backslash \overline{P S O}$ such that $h \mid I$ is $C^{1}$.

Proof. Since $h$ is absolutely continuous, $h^{\prime}$ exists a.e. in $M$ and is integrable and $h=\int h^{\prime}(x) d x$ (refer to $[\mathbf{A B}, \mathbf{V E}]$ ). Let $\Lambda=$ $\cup_{n=0}^{\infty} f^{-n}(\overline{P S O})$. Then $m(\Lambda)=0$ since $\overline{P S O}$ has zero measure. Since $h^{\prime}(x)$ is measurable and $h$ is a homeomorphism, we can find a point $p$ in $M \backslash \Lambda$ and a subset $p \in E_{0}$ such that $h$ is differentiable at every point in $E_{0}$, such that $p$ is a density point of $E_{0}$, such that $h^{\prime}(p) \neq 0$, and such that the derivative $h^{\prime} \mid E_{0}$ is continuous at $p$ (refer to $[\mathbf{A B}, \mathbf{V E}]$ ). Since $f$ is nice, there is a subsequence $\left\{f^{\circ n_{k}}(p)\right\}_{k=1}^{\infty} \subseteq M \backslash W$ such that it converges to a point $q$ in $M \backslash W$, where $W$ is the open set in $(I I)$ in the beginning of $\S 4$.

Let us first assume that $q$ is an interior point of $U$. Take a small interval $q \in I=(a, b) \subset U$. Assume $\left\{f^{\circ n_{k}}(p)\right\}_{k=1}^{\infty} \subset I$. Since $I \cap$ $\overline{P S O}=\emptyset$, there is a sequence of interval $\left\{J_{k}\right\}_{k=1}^{\infty}$ such that $p \in J_{k}$, such that $f^{\circ n_{k}}: J_{k} \rightarrow I$ is a $C^{1}$-diffeomorphism, and such that $\left\{J_{l, k}=\right.$ $\left.f^{\circ l}\left(J_{k}\right)\right\}_{l=0}^{n_{k}}$ is admissible for every $k \geq 1$. From $(i i)$ of Definition 2, $m\left(J_{k}\right)$ goes to zero as $k$ tends to infinity.

From Lemma 2, there is a constant $C>0$, such that

$$
\left|\log \left(\frac{\left|\left(f^{\circ n_{k}}\right)^{\prime}(w)\right|}{\left|\left(f^{\circ n_{k}}\right)^{\prime}(z)\right|}\right)\right| \leq C
$$

for any $w$ and $z$ in $J_{k}$. (The inequality means that $\left\{f^{\circ n_{k}} \mid J_{k}\right\}_{k=1}^{\infty}$ have uniformly bounded distortion.) For any positive integer $s$, there is an 
integer $N_{s}>0$ such that

$$
\frac{m\left(E_{0} \cap J_{k}\right)}{m\left(J_{k}\right)} \geq 1-\frac{1}{s}
$$

for all $k>N_{s}$. Let $E_{k}=f^{\circ n_{k}}\left(E_{0} \cap J_{k}\right)$. Then $h$ is differentiable at every point in $E_{k}$ and

$$
\frac{m\left(E_{k} \cap I\right)}{m(I)} \geq 1-\frac{C}{s}
$$

for all $k>N_{s}$ because $\left\{f^{\circ n_{k}} \mid J_{k}\right\}_{k=1}^{\infty}$ have uniformly bounded distortion where $C>0$ is a constant. Let $E=\cap_{s=1}^{\infty} \cup_{k>N_{s}} E_{k}$. Then $E$ has full measure in $I$ and $h$ is differentiable at every point in $E$ with non-zero derivative.

Next, we are going to prove that $h^{\prime} \mid E$ is uniformly continuous. For any $x$ and $y$ in $E$, let $z_{k}$ and $w_{k}$ be the preimages of $x$ and $y$ under the diffeomorphism $f^{\circ n_{k}}: J_{k} \rightarrow I$. Then $z_{k}$ and $w_{k}$ are in $E_{0}$. From $h \circ f=g \circ h$, we have that

$$
h^{\prime}(x)=\frac{\left(g^{\circ n_{k}}\right)^{\prime}\left(h\left(z_{k}\right)\right)}{\left(f^{\circ n_{k}}\right)^{\prime}\left(z_{k}\right)} h^{\prime}\left(z_{k}\right)
$$

and

$$
h^{\prime}(y)=\frac{\left(g^{\circ n_{k}}\right)^{\prime}\left(h\left(w_{k}\right)\right)}{\left(f^{\circ n_{k}}\right)^{\prime}\left(w_{k}\right)} h^{\prime}\left(w_{k}\right) .
$$

So

$$
\begin{aligned}
\left|\log \left(\frac{h^{\prime}(x)}{h^{\prime}(y)}\right)\right| & \leq|\log | \frac{\left(g^{\circ n_{k}}\right)^{\prime}\left(h\left(z_{k}\right)\right)}{\left(g^{\circ n_{k}}\right)^{\prime}\left(h\left(w_{k}\right)\right)}||+|\log | \frac{\left(f^{\circ n_{k}}\right)^{\prime}\left(w_{k}\right)}{\left(f^{\circ n_{k}}\right)^{\prime}\left(z_{k}\right)}|| \\
& +\left|\log \left(\frac{h^{\prime}\left(z_{k}\right)}{h^{\prime}\left(w_{k}\right)}\right)\right|
\end{aligned}
$$

From Lemma 2, there is a constant $C>0$ such that

$$
|\log | \frac{\left(f^{\circ n_{k}}\right)^{\prime}\left(w_{k}\right)}{\left(f^{\circ n_{k}}\right)^{\prime}\left(z_{k}\right)}|| \leq C|x-y|^{\alpha}
$$

and

Therefore,

$$
|\log | \frac{\left(g^{\circ n_{k}}\right)^{\prime}\left(h\left(z_{k}\right)\right)}{\left(g^{\circ n_{k}}\right)^{\prime}\left(h\left(w_{k}\right)\right)}|| \leq C|h(x)-h(y)|^{\alpha} .
$$

$$
\left|\log \left(\frac{h^{\prime}(x)}{h^{\prime}(y)}\right)\right| \leq C\left(|x-y|^{\alpha}+|h(x)-h(y)|^{\alpha}\right)+\left|\log \left(\frac{h^{\prime}\left(z_{k}\right)}{h^{\prime}\left(w_{k}\right)}\right)\right| .
$$


Since $h^{\prime} \mid E_{0}$ is continuous at $p$, the last term in the last inequality tends to zero as $k$ goes to infinity. Hence

$$
\left|\log \left(\frac{h^{\prime}(x)}{h^{\prime}(y)}\right)\right| \leq C\left(|x-y|^{\alpha}+|h(x)-h(y)|^{\alpha}\right) .
$$

This means that $h^{\prime} \mid E$ is uniformly continuous. It can be extended to a continuous function $\phi$ on $I$. Because $h \mid I$ is absolutely continuous and $E$ has full measure in $I$,

$$
h(x)=h(a)+\int_{a}^{x} h^{\prime}(x) d x=h(a)+\int_{a}^{x} \phi(x) d x
$$

on $I$. This implies that $h \mid I$ is actually $C^{1}$.

If $q$ is in $V$ but not an interior point of $U$. We can find a small interval $I=(a, b)$ such that $q \in I \subseteq V$ and such that $m(I)=b-a \leq$ $\operatorname{dist}(I, \overline{P S O})$ and $m(h(I)) \leq \operatorname{dist}(h(I), \overline{h(P S O)})$ where dist means the distance. Modifying the above argument either by only applying Lemma 1 or by first applying Lemma 1 and then Lemma 2, we can still prove that $h \mid I$ is $C^{1}$ (refer to the proof of Lemma 5).

Remember that $U_{i}(\tau)=\left[c_{i}-\tau, c_{i}+\tau\right]$ for $c_{i} \in C P$. Let $I$ and $q$ be the interval and the point found in the previous lemma.

LEMMA 4. If $h$ is absolutely continuous, then the restriction of $h$ to every $U_{i}(\tau)$ is $C^{1+\alpha}$.

Proof. Denote $J=U_{i}(\tau)=[d, e]$. Since $f$ is mixing and since $J \cap \overline{P S O}=\emptyset$, there is a preimage $J_{k} \subset I$ of $J$ under $f^{\circ n_{k}}$ such that $J_{k}$ tends to $q$ as $k \rightarrow \infty$ and such that $f^{\circ n_{k}}: J_{k} \rightarrow J$ is a $C^{1}$ diffeomorphism where $\left\{n_{k}\right\}_{k=1}^{\infty}$ is a subset of the positive integers. Let $F_{k}$ be the inverse of $f^{\circ n_{k}}: J_{k} \rightarrow J$ for all $k>0$. From the equation $h \circ f=g \circ h$, we have that $h \mid J=g^{\circ n_{k}} \circ h \circ F_{k}$. So $h \mid J$ is $C^{1}$ and

$$
h^{\prime}(x)=\frac{\left(g^{\circ n_{k}}\right)^{\prime}\left(h\left(z_{k}\right)\right)}{\left(f^{\circ n_{k}}\right)^{\prime}\left(z_{k}\right)} h^{\prime}\left(z_{k}\right) \neq 0
$$

for any $x$ in $J$ where $z_{k}=F_{k}(x)$.

Without loss of generality, we assume that $\left\{J_{i, k}=f^{\circ\left(n_{k}-i\right)}\left(J_{k}\right)\right\}_{i=0}^{n_{k}}$ are all admissible for all $k>0$. For any $x$ and $y$ in $J$, let $z_{k}$ and $w_{k}$ be the preimage of $x$ and $y$ under the diffeomorphism $f^{\circ n_{k}}: J_{k} \rightarrow J$. Since

$$
\frac{h^{\prime}(x)}{h^{\prime}(y)}=\frac{\left(g^{\circ n_{k}}\right)^{\prime}\left(h\left(z_{k}\right)\right)}{\left(f^{\circ n_{k}}\right)^{\prime}\left(z_{k}\right)} \cdot \frac{\left(f^{\circ n_{k}}\right)^{\prime}\left(w_{k}\right)}{\left(g^{\circ n_{k}}\right)^{\prime}\left(h\left(w_{k}\right)\right)} \cdot \frac{h^{\prime}\left(z_{k}\right)}{h^{\prime}\left(w_{k}\right)},
$$


from Lemma 2, there is a constant $C>0$ such that

$$
\begin{aligned}
\left|\log \left(\frac{h^{\prime}(x)}{h^{\prime}(y)}\right)\right| & \leq|\log | \frac{\left(g^{\circ n_{k}}\right)^{\prime}\left(h\left(z_{k}\right)\right)}{\left(g^{\circ n_{k}}\right)^{\prime}\left(h\left(w_{k}\right)\right)}||+|\log | \frac{\left(f^{\circ n_{k}}\right)^{\prime}\left(w_{k}\right)}{\left(f^{\circ n_{k}}\right)^{\prime}\left(z_{k}\right)}|| \\
& +\left|\log \left(\frac{h^{\prime}\left(z_{k}\right)}{h^{\prime}\left(w_{k}\right)}\right)\right| \\
& \leq C\left(|x-y|^{\alpha}+|h(x)-h(y)|^{\alpha}\right)+\left|\log \left(\frac{h^{\prime}\left(z_{k}\right)}{h^{\prime}\left(w_{k}\right)}\right)\right| .
\end{aligned}
$$

Because $h^{\prime}\left(z_{k}\right), h^{\prime}\left(w_{k}\right) \rightarrow h^{\prime}(q)$ as $k \rightarrow \infty$, we have that

$$
\left|\log \left(\frac{h^{\prime}(x)}{h^{\prime}(y)}\right)\right| \leq C\left(|x-y|^{\alpha}+|h(x)-h(y)|^{\alpha}\right) .
$$

This implies that $h \mid J$ is actually $C^{1+\alpha}$.

LEMMA 5. If $h$ is absolutely continuous and if the exponents of $f$ and $g$ at all singular points are the same, then the restriction of $h$ to the closure of every interval $J$ of $V \backslash \overline{P S O}$ is $C^{1+\frac{\alpha}{\gamma}}$.

Proof. We always use $C$ to denote a positive constant (although it may be different in different formulas). Since $f$ is mixing, we can find a subsequence $\left\{n_{k}\right\}_{k=1}^{\infty}$ of the positive integers and intervals $J_{k} \subset$ $I$ such that $J_{k}$ tends to $q$ as $k \rightarrow \infty$ and such that $f^{\circ n_{k}}: J_{k} \rightarrow J$ is a diffeomorphism. Let $F_{k}$ be the inverse of $f^{\circ n_{k}}: J_{k} \rightarrow J$. From the equation $h \circ f=g \circ h$, we have that $h \mid J=g^{\circ n_{k}} \circ h \circ F_{k}$. So $h \mid J$ is $C^{1}$ and

$$
h^{\prime}(x)=\frac{\left(g^{\circ n_{k}}\right)^{\prime}\left(h\left(z_{k}\right)\right)}{\left(f^{\circ n_{k}}\right)^{\prime}\left(z_{k}\right)} h^{\prime}\left(z_{k}\right) \neq 0
$$

for any $x$ in $J$ where $z_{k}=F_{k}(x)$.

Let $J_{i, k}=f^{\circ\left(n_{k}-i\right)}\left(J_{k}\right)$ for $0 \leq i \leq n_{k}$. Without loss of generality, we assume that $\left\{J_{i, k}\right\}_{i=0}^{n_{k}}$ is admissible. For any $x$ and $y$ in $J$, let $z_{k}$ and $w_{k}$ be the preimage of $x$ and $y$ under the diffeomorphism $f^{\circ n_{k}}: J_{k} \rightarrow J$. Since

$$
\begin{aligned}
\frac{h^{\prime}(x)}{h^{\prime}(y)}=\frac{\left(g^{\circ n_{k}}\right)^{\prime}\left(h\left(z_{k}\right)\right)}{\left(f^{\circ n_{k}}\right)^{\prime}\left(w_{k}\right)} \cdot \frac{\left(f^{\circ n_{k}}\right)^{\prime}\left(w_{k}\right)}{\left(g^{\circ n_{k}}\right)^{\prime}\left(h\left(w_{k}\right)\right)} \cdot \frac{h^{\prime}\left(z_{k}\right)}{h^{\prime}\left(w_{k}\right)}, \\
\left|\log \left(\frac{h^{\prime}(x)}{h^{\prime}(y)}\right)\right| \leq\left|\log \left(\frac{\left|\left(g^{\circ n_{k}}\right)^{\prime}\left(h\left(z_{k}\right)\right)\right|}{\left|\left(g^{\circ n_{k}}\right)^{\prime}\left(h\left(w_{k}\right)\right)\right|}\right)\right| \\
+\left|\log \left(\frac{\left|\left(f^{\circ n_{k}}\right)^{\prime}\left(w_{k}\right)\right|}{\left|\left(f^{\circ n_{k}}\right)^{\prime}\left(z_{k}\right)\right|}\right)\right|+\left|\log \left(\frac{h^{\prime}\left(z_{k}\right)}{h^{\prime}\left(w_{k}\right)}\right)\right| .
\end{aligned}
$$


Let $n=n_{k}$ and let $m=m\left(n_{k}\right)>0$ be the smallest integer such that $J_{m, k} \subseteq U_{j}(\tau) \subseteq U$ for some $1 \leq j \leq d$. Let $x_{i}=f^{\circ\left(n_{k}-i\right)}\left(z_{k}\right)$ and $y_{i}=f^{\circ\left(n_{k}-i\right)}\left(w_{k}\right)$ for all $0 \leq i \leq n_{k}$. Then

$$
\begin{aligned}
\left|\log \left(\frac{h^{\prime}(x)}{h^{\prime}(y)}\right)\right| & \leq\left|\sum_{i=1}^{m-1}\left(\log \left|f^{\prime}\left(y_{i}\right)\right|-\log \left|f^{\prime}\left(x_{i}\right)\right|\right)\right| \\
& +\left|\sum_{i=1}^{m-1}\left(\log \left|g^{\prime}\left(h\left(x_{i}\right)\right)\right|-\log \left|g^{\prime}\left(h\left(y_{i}\right)\right)\right|\right)\right| \\
& +\left|\log \left(\frac{\left|g^{\prime}\left(h\left(x_{m}\right)\right)\right|}{\left|f^{\prime}\left(x_{m}\right)\right|} \cdot \frac{\left|f^{\prime}\left(y_{m}\right)\right|}{\left|g^{\prime}\left(h\left(y_{m}\right)\right)\right|}\right)\right| \\
& +\left|\sum_{i=m+1}^{n}\left(\log \left|f^{\prime}\left(y_{i}\right)\right|-\log \left|f^{\prime}\left(x_{i}\right)\right|\right)\right| \\
& +\left|\sum_{i=m+1}^{n}\left(\log \left|g^{\prime}\left(h\left(x_{i}\right)\right)\right|-\log \left|g^{\prime}\left(h\left(y_{i}\right)\right)\right|\right)\right| \\
& +\left|\log \left(\frac{h^{\prime}\left(z_{k}\right)}{h^{\prime}\left(w_{k}\right)}\right)\right| .
\end{aligned}
$$

The last term tends to zero as $k$ goes to infinity since $h^{\prime}\left(w_{k}\right), h^{\prime}\left(z_{k}\right) \rightarrow$ $h^{\prime}(q)$ as $k \rightarrow \infty$. We estimate the first five terms. From Lemma 1, there is a constant $C>0$ such that

$$
\left|\sum_{i=1}^{m-1}\left(\log \left|f^{\prime}\left(y_{i}\right)\right|-\log \left|f^{\prime}\left(x_{i}\right)\right|\right)\right| \leq C|x-y|^{\alpha}
$$

and

$$
\left|\sum_{i=1}^{m-1}\left(\log \left|g^{\prime}\left(h\left(x_{i}\right)\right)\right|-\log \left|g^{\prime}\left(h\left(y_{i}\right)\right)\right|\right)\right| \leq C|h(x)-h(y)|^{\alpha} .
$$

From Lemma 2, there is a constant $C>0$ such that

$$
\begin{aligned}
\left|\sum_{i=m+1}^{n}\left(\log \left|f^{\prime}\left(y_{i}\right)\right|-\log \left|f^{\prime}\left(x_{i}\right)\right|\right)\right| & \leq C\left|x_{m}-y_{m}\right|^{\alpha} \\
& \leq C\left|x_{m-1}-y_{m-1}\right|^{\frac{\alpha}{\gamma_{j}}} \\
& \leq C|x-y|^{\frac{\alpha}{\gamma_{j}}} .
\end{aligned}
$$


Similarly,

$$
\begin{aligned}
\left|\sum_{i=m+1}^{n}\left(\log \left|g^{\prime}\left(h\left(x_{i}\right)\right)\right|-\log \left|g^{\prime}\left(h\left(y_{i}\right)\right)\right|\right)\right| & \leq C\left|h\left(x_{m}\right)-h\left(y_{m}\right)\right|^{\frac{\alpha}{\gamma_{j}}} \\
& \leq C|h(x)-h(y)|^{\frac{\alpha}{\gamma_{j}}} .
\end{aligned}
$$

Now we consider

$$
\mathcal{S}=\frac{\left|g^{\prime}\left(h\left(x_{m}\right)\right)\right|}{\left|f^{\prime}\left(x_{m}\right)\right|} \cdot \frac{\left|f^{\prime}\left(y_{m}\right)\right|}{\left|g^{\prime}\left(h\left(y_{m}\right)\right)\right|} .
$$

Define

$$
\mathcal{S}=\mathcal{S}_{1} \cdot \mathcal{S}_{2} \cdot \mathcal{S}_{3}
$$

where

$$
\begin{gathered}
\mathcal{S}_{1}=\frac{\left|g^{\prime}\left(h\left(x_{m}\right)\right)\right|}{\left|h\left(x_{m}\right)-h\left(c_{j}\right)\right|^{\gamma_{j}-1}} \cdot \frac{\left|h\left(y_{m}\right)-h\left(c_{j}\right)\right|^{\gamma_{j}-1}}{\mid g^{\prime}\left(h\left(y_{m}\right)\right)}, \\
\mathcal{S}_{2}=\frac{\left|x_{m}-c_{j}\right|^{\gamma_{j}-1}}{\left|f^{\prime}\left(x_{m}\right)\right|} \cdot \frac{\left|f^{\prime}\left(y_{m}\right)\right|}{\left|y_{m}-c_{j}\right|^{\gamma_{j}-1}},
\end{gathered}
$$

and

$$
\mathcal{S}_{3}=\left(\frac{\left|h\left(x_{m}\right)-h\left(c_{j}\right)\right|}{\left|x_{m}-c_{j}\right|}\right)^{\gamma_{j}-1} \cdot\left(\frac{\left|y_{m}-c_{j}\right|}{\left|h\left(y_{m}\right)-h\left(c_{j}\right)\right|}\right)^{\gamma_{j}-1} .
$$

Lemma 4 implies that

$$
\left|\log S_{3}\right| \leq C\left|x_{m}-y_{m}\right|^{\alpha} \leq C\left|x_{m-1}-y_{m-1}\right|^{\frac{\alpha}{\gamma_{j}}} \leq C|x-y|^{\frac{\alpha}{\gamma_{j}}} .
$$

From (1) of Definition 2,

$$
\left|\log S_{2}\right| \leq C\left|x_{m}-y_{m}\right|^{\alpha} \leq C\left|x_{m-1}-y_{m-1}\right|^{\frac{\alpha}{\gamma_{j}}} \leq C|x-y|^{\frac{\alpha}{\gamma_{j}}}
$$

and

$$
\begin{aligned}
\left|\log S_{1}\right| & \leq C\left|h\left(x_{m}\right)-h\left(y_{m}\right)\right|^{\alpha} \leq C\left|h\left(x_{m-1}\right)-h\left(y_{m-1}\right)\right|^{\frac{\alpha}{\gamma_{j}}} \\
& \leq C|h(x)-h(y)|^{\frac{\alpha}{\gamma_{j}}} .
\end{aligned}
$$

Thus,

$$
\left|\log \left(\frac{h^{\prime}(x)}{h^{\prime}(y)}\right)\right| \leq C\left(|x-y|^{\frac{\alpha}{\gamma}}+|h(x)-h(y)|^{\frac{\alpha}{\gamma}}\right) .
$$

This implies that $h^{\prime} \mid J$ is actually $C^{\frac{\alpha}{\gamma}}$. So is $h^{\prime} \mid \bar{J}$. We have that $h \mid \bar{J}$ is $C^{1+\frac{\alpha}{\gamma}}$. 
Proof of the Main Theorem. Lemmas 4 and 5 and Remark 3 and the calculation before Remark 3 complete the proof.

\section{Geometrically finite one-dimensional maps}

Let $M$ be the interval $[-1,1]$ or $\mathbb{S}^{1}$. Let $f: M \rightarrow M$ be a piecewise $C^{1}$ map with only power law type singular points. Let $S P$ be the set of singular points of $f$ and $S O=\cup_{n=0}^{\infty} f^{\circ n}(S P)$ be the union of singular orbits of $f$. If $S O$ is non-empty and finite, let $\xi_{1}=\left\{I_{0}, \cdots, I_{k-1}\right\}$ be the set of the closures of intervals in the complement of $S O$ in $M$, then $\left(f, \xi_{1}\right)$ is a Markov map. By a Markov map, we mean that

a. $I_{0}, \ldots, I_{k-1}$ have pairwise disjoint interiors,

b. the union $\cup_{i=0}^{k-1} I_{i}$ of all intervals in $\xi_{1}$ is $M$,

c. the restriction $f \mid I$ to every interval $I$ in $\xi_{1}$ is homeomorphic, and

d. the image $f(I)$ of every interval $I$ in $\xi_{1}$ is the union of some intervals in $\xi_{1}$.

Suppose $\left(f, \xi_{1}\right)$ is the Markov map in the previous paragraph. Let $g_{i}=\left(f \mid I_{i}\right)^{-1}$ be the inverse of $f: I_{i} \rightarrow f\left(I_{i}\right)$ for $0 \leq i<k$. A sequence $w_{n}=i_{0} \cdots i_{n-1}$ of 0 's, $\cdots,(k-1)$ 's is called admissible if the domain $f\left(I_{i_{l}}\right)$ of $g_{i_{l}}$ contains $I_{i_{l+1}}$ for all $0 \leq l<n-1$. For an admissible sequence $w_{n}=i_{0} \cdots i_{n-1}$ of 0 's, $\cdots,(k-1)$ 's, we can define $g_{w_{n}}=g_{i_{0}} \circ \cdots g_{i_{n-1}}$ and $I_{w_{n}}=g_{w_{n}}\left(f\left(I_{i_{n-1}}\right)\right)$. Let $\xi_{n}$ be the set of the intervals $I_{w_{n}}$ for all admissible sequences of length $n$. It is also a Markov partition of $M$ respect to $f$ and called the $n^{t h}$-partition of $M$ induced from $\left(f, \xi_{1}\right)$. Let $\kappa_{n}$ be the maximum of the lengths of intervals in $\xi_{n}$. A one-dimensional map $f: M \rightarrow M$ is said to be geometrically finite if

(1) $f$ is $C^{1+}$,

(2) the set of singular orbits $S O$ is non-empty and finite,

(3) no critical point is periodic, and

(4) there are constants $C>0$ and $0<\mu<1$ such that $\kappa_{n} \leq C \mu^{n}$ for all $n>0$.

Suppose $f$ is a geometrically finite one-dimensional map. It is quasi-hyperbolic from $[\mathbf{J I 3}, \mathbf{J I 5}]$. The set of post-singular orbits $P S O$ of $f$ is finite, so it has zero measure, i.e., $m(\overline{P S O})=0$. Every singular point $u$ of $f$ lands at an expanding periodic orbit of $f$, this means that there is an integer $k>0$ such that $v=f^{\circ k}(u)$ is an expanding periodic point of $f$ (see $[\mathbf{J I 3}, \mathbf{J I 5}])$. Therefore $f$ is nice. Let $F S P$ be the set 
of all fold singular points of $f$ and let $P F S O=\cup_{n=1}^{\infty} f^{\circ n}(F S P)$ be the set of all post fold singular orbits of $f$ (see the beginning of $\S 4$ ). Then PFSO is finite. Let $M_{0}=M \backslash P F S O$. Let $\eta$ be the set of the closures of intervals of $M \backslash P F S O$. It is finite. We have that

Corollary 1. Suppose $f$ and $g$ are two conjugate mixing geometrically finite one-dimensional maps. Let $h$ be the conjugacy between $f$ and $g$, i.e., $h \circ f=g \circ h$. Suppose $h$ is orientation-preserving. Then $h \mid I$ for any $I$ in $\eta$ is a $C^{1+\beta}$-diffeomorphism for some $0<\beta \leq 1$ if and only if (i) $h$ is an absolutely continuous homeomorphism and (ii) the exponents and the asymmetries of $f$ and $g$ at all corresponding critical points are the same.

REMARK 4. For an orientation-preserving conjugacy class $\mathcal{F}$ of geometrically finite one-dimensional maps, there is the topological model: the symbolic space $\Sigma$ with the shift map $\sigma$. This topological model is called the symbolic dynamical system for $\mathcal{F}$ (see [JI5]). The dual symbolic space $\Sigma^{*}$ of $\mathcal{F}$ and the scaling function

$$
s_{f}: \Sigma^{*} \rightarrow \mathbb{R}
$$

of $f$ in $\mathcal{F}$ were defined in [JI5]. The scaling function $s_{f}$ of $f$ is a smooth invariant in $\mathcal{F}$. We proved in [JI5] that the scaling function $s_{f}$ exists for every geometrically finite one-dimensional map. The property of the scaling function $s_{f}$ of a geometrically finite one-dimensional map $f$ has been also studied in details in [JI5]. Furthermore, the scaling function is used as a complete smooth invariant along with exponents and asymmetries in the smooth classification of geometrically finite one-dimensional maps. We proved in [JI5] that if the scaling functions $s_{f}$ and $s_{g}$ of $f$ and $g$ in $\mathcal{F}$ are the same, the conjugacy $h$ between $f$ and $g$ is bi-Lipschitz, and further, if the exponents and asymmetries of $f$ and $g$ at corresponding singular points are also the same, then $h$ restricted on every interval in $\eta$ is a $C^{1+\beta}$-diffeomorphism. The analysis from bi-Lipschitz to $C^{1+\beta}$ in $[\mathbf{J I 5}]$ is not quite complete. However, a bi-Lipschitz homeomorphism is an absolutely continuous homeomorphism, it can be closed by Corollary 1 .

\section{Generalised Ulam-von Neumann transformations}

Let $M=[-1,1]$ and let $f: M \rightarrow M$ be a $C^{1+}$-folding map with a unique power law type singular point 0 . We call $f: M \rightarrow M$ a generalised Ulam-von Neumann transformation (see $[\mathbf{J I 1}, \mathbf{J I 4}]$ ) if

[a] $f(-1)=f(1)=-1$ and $f(0)=1$, 
[b] $f \mid[-1,0]$ and $f \mid[0,1]$ are orientation-preserving and orientationreversing homeomorphisms, and

[c] $f$ is a geometrically finite one-dimensional map.

One example of a generalised Ulam-von Neumann transformation is $f(x)=1-2|x|^{\gamma}$ for $\gamma>1$. Another one is $g(x)=-1+2 \cos (\pi x / 2)$. For a generalised Ulam-von Neumann transformation $f$, let $I_{0}=$ $[-1,0]$ and $I_{1}=[0,1]$. We then have that $f\left(I_{0}\right)=f\left(I_{1}\right)=M$. Thus $\xi_{1}=\left\{I_{0}, I_{1}\right\}$ is a Markov partition of $M$ with respect to $f$. From $[\mathbf{J I 1}, \mathbf{J I 4}]$ a generalised Ulam-von Neumann transformation $f$ is a nice and mixing quasi-hyperbolic one-dimensional map. The postsingular orbit $P S O=\cup_{i=1}^{\infty} f^{\circ i}(0)$ of $f$ is the boundary $\{-1,1\}$ of $M$. Any two generalised Ulam-von Neumann transformations $f$ and $g$ are topologically conjugate by an orientation-preserving homeomorphism $h$ (see $[\mathbf{J I} 4]$ ). Following the Main Theorem, we have that

Corollary 2. Let $f$ and $g$ be two generalised Ulam-von Neumann transformations. Let $h$ be the conjugacy from $f$ to $g$, i.e., $h \circ f=g \circ h$. Then $h$ is a $C^{1+\beta}$-diffeomorphism for some $0<\beta \leq 1$ if and only if $h$ is an absolutely continuous homeomorphism and the exponents and the asymmetries of $f$ and $g$ at 0 are the same.

If the eigenvalues (refer to $\S 2$ ) at corresponding periodic points and the exponents at 0 of two generalised Ulam-von Neumann transformations of $f$ and $g$ are the same, then $h$ is a bi-Lipschitz homeomorphism (see [JI4]). So $h$ is an absolutely continuous homeomorphism in this case. In [JI4], we argued from bi-Lipschitz to $C^{1}$ by the Gibbs theory. Now it can follow from the Main Theorem.

Corollary 3. Let $f$ and $g$ be two generalised Ulam-von Neumann transformations. Let $h$ be the conjugacy from $f$ to $g$, i.e., $h \circ f=g \circ h$. Then $h$ is a $C^{1+\beta}$-diffeomorphism for some $0<\beta \leq 1$ if and only if the eigenvalues of $f$ and $g$ at corresponding periodic points and the exponents and asymmetries of $f$ and $g$ at 0 are the same.

REMARK 5. Let $f: M \rightarrow M$ be a generalised Ulam-von Neumann transformation. For any $x \in M$, we can find a unique sequence $\left\{x_{i}\right\}_{i=1}^{\infty} \subset[-1,0]$ such that $f\left(x_{i}\right)=x_{i-1}$ for $1 \leq i<\infty$ where $x_{0}=x$. One can check that $x_{i} \rightarrow-1$ as $i \rightarrow \infty$. Let $g: M \rightarrow M$ be another generalised Ulam-von Neumann transformation and let $h$ be the conjugacy from $f$ to $g$, i.e., $h \circ f=g \circ h$. If $h$ is a $C^{1}$-diffeomorphism, we 
have that

$$
h^{\prime}(x)=\left(\prod_{i=1}^{\infty} \frac{g^{\prime}\left(h\left(x_{i}\right)\right)}{f^{\prime}\left(x_{i}\right)}\right) \cdot h^{\prime}(-1) .
$$

Using this equality, one can discuss the $C^{k+\beta}$ (or real analytic) smoothness of $h$ in Corollaries 2 and 3 if both $f$ and $g$ are $C^{k+\alpha}$ in the sense of Definition 1 (or real analytic) where $k \geq 1$ is an integer and $0<\beta \leq \alpha \leq 1$ are real numbers.

\section{Expanding circle endomorphisms}

Let $\mathbb{S}^{1}=\{z \in \mathbb{C}|| z \mid=1\}$ be the unit circle. Let $f: \mathbb{S}^{1} \rightarrow \mathbb{S}^{1}$ be a $C^{1+\alpha}$-orientation-preserving endomorphism for some $0<\alpha \leq 1$. We say $f$ is expanding if there are constants $C>0$ and $\mu>1$ such that $\left|\left(f^{\circ n}\right)^{\prime}(x)\right| \geq C \mu^{n}$ for all $x \in \mathbb{S}^{1}$ and all $n \geq 1$. Let $d=\operatorname{deg}(f)$ be the topological degree of $f$. An example of an expanding circle endomorphism of degree $d$ is $z \mapsto z^{d}$. Shub $[\mathbf{S H}]$ proved that every degree $d$ such map is topologically conjugate to $z \mapsto z^{d}$. Thus any two $C^{1+\alpha}$-orientation-preserving expanding circle endomorphisms $f$ and $g$ of degree $d>1$ are topologically conjugate by an orientationpreserving homeomorphism $h$ of $\mathbb{S}^{1}$. The following result is due to Sullivan [SU3] and can now follow from the Main Theorem.

Corollary 4 (Sullivan). Let $f$ and $g$ be two $C^{1+\alpha}$-orientationpreserving expanding circle endomorphisms of degree $d>1$ where $0<\alpha \leq 1$ is a real number. Let $h$ be the conjugacy from $f$ to $g$, i.e., $h \circ f=g \circ h$. Then $h$ is a $C^{1+\alpha}$-diffeomorphism if and only if the eigenvalues of $f$ and $g$ at all periodic points of $f$ are the same.

REMARK 6. A $C^{1}$-orientation-preserving expanding circle endomorphism $f: \mathbb{S}^{1} \rightarrow \mathbb{S}^{1}$ of degree $d>1$ has a fixed point $q$. The preimage $f^{-1}(q)$ contains $d$ points, and $\mathbb{S}^{1} \backslash f^{-1}(q)$ has $d$ connected components. Let $I_{0}$ be the closure of a fixed interval of $\mathbb{S}^{1} \backslash f^{-1}(q)$ having $q$ as an endpoint. For any $x \in \mathbb{S}^{1}$, we can find a unique sequence $\left\{x_{i}\right\}_{i=1}^{\infty} \subset I_{0}$ such that $f\left(x_{i}\right)=x_{i-1}$ for $1 \leq i<\infty$ where $x_{0}=x$. One can check that $x_{i} \rightarrow q$ as $i \rightarrow \infty$. Let $g: \mathbb{S}^{1} \rightarrow \mathbb{S}^{1}$ be another $C^{1}$-orientation-preserving expanding circle endomorphism of degree $d$ and let $h$ be the conjugacy from $f$ to $g$, i.e., $h \circ f=g \circ h$. If $h$ is a $C^{1}$-diffeomorphism, then

$$
h^{\prime}(x)=\left(\prod_{i=1}^{\infty} \frac{g^{\prime}\left(h\left(x_{i}\right)\right)}{f^{\prime}\left(x_{i}\right)}\right) \cdot h^{\prime}(q) .
$$


Using this equality, one can discuss the $C^{k+\alpha}$ smoothness of $h$ in Corollary 4 if both $f$ and $g$ are $C^{k+\alpha}$ where $k>1$ is an integer and $0<\alpha \leq 1$ is a real number. In particular, if $f$ and $g$ in Corollary 4 are both real analytic, then $h$ is real analytic (see $[\mathbf{S S}]$ ).

\section{References}

[BO] R. Bowen, Hausdorff Dimension of Quasi-circles, Publ. of IHES 50 (1979).

[AB] A. M., Bruckner, Differentiation of Real Functions, Lecture Notes in Mathematics 659 (1978), Springer-Verlag, Berlin, New York, 18-19.

[VE] V. Ene, Real Functions-Current Topics, Lecture Notes in Mathematics 1603 (1995), Springer-Verlag, Berlin, New York, 14.

[GS1] F. Gardiner and D. Sullivan, Lacunary series as quadratic differentials in conformal dynamics, Contemporary Mathematics 169 (1994), 307-330.

[GS2] F. Gardiner and D. Sullivan, Symmetric and quasisymmetric structures on a closed curve, Amer. J. of Math. 114, no. 4 (1992), 683-736.

[JI1] Y. Jiang, Generalized Ulam-von Neumann transformations, Thesis, the Graduate Center of CUNY (1990).

[JI2] Y. Jiang, Dynamics of certain one-dimensional maps, I. $C^{1+\alpha}$-DenjoyKoebe distortion lemma, IMS preprint 1/1991, SUNY at Stony Brook (1991).

[JI3] Y. Jiang, Geometry of geometrically finite one-dimensional maps, Commun. in Math. Phys. 156 (1993), 639-647.

[JI4] Y. Jiang, On Ulam-von Neumann transformations, Commun. in Math. Phys. 172 (1995), 449-459.

[JI5] Y. Jiang, Smooth classification of geometrically finite one-dimensional maps, The Trans. of the Amer. Math. Soc. 348, No. 6 (June, 1996), 2391-2412..

[MV] W. de Melo and S. van Strien, One-Dimensional Dynamics (1993), SpringerVerlag, Berlin, New York.

[MC1] C. McMullen, Complex Dynamics and Renormalization 135 (1994), Ann. of Math. Stud., Princeton, NJ.

[MC2] C. McMullen, Renormalization and 3-manifolds which fiber over the circle, Preprint (1995).

[MO] D. Mostow, Strong rigidity of locally symmetric spaces 78 (1972), Ann. of Math. Stud., Princeton, NJ.

[SH] M. Shub, Endomorphisms of compact differentiable manifolds, Amer. J. Math. 91 (1969), 129-155.

[SS] M. Shub and D. Sullivan, Expanding Endomorphisms of the circle revisited, Ergod. Th \& Dynam. Sys. 5 (1987), 285-289.

[SU1] D. Sullivan, Bounds, quadratic differentials, and renormalization conjectures, Amer. Math. Soc. Centennial Publications 2 (1992), 417-466.

[SU2] D. Sullivan, The density at infinity of a discrete group of hyperbolic isometries, Publ. Math. of IHES 50 (1979), 171-202.

[SU3] D. Sullivan, Private communications (1987).

[TH] W. Thurston, Geometry and topology of three-manifolds, Preprint (1979), Princeton University. 
[TU] P. Tukia, Differentiability and rigidity of Möbius groups, Invent. Math. 82 (1985), 557-578.

Yunping Jiang: Department of Mathematics, Queens College of CUNY, Flushing, NY 11367-1597, U.S.A.

E-mail address: yunqc@yunping.math.qc.edu 